\title{
Droplet pattern and condensation gradient around a humidity sink
}

\author{
J. Guadarrama-Cetina,,${ }^{1, *}$ R. D. Narhe, ${ }^{1}$ D. A. Beysens,${ }^{2,3}$ and W. González-Viñas ${ }^{1, \dagger}$ \\ ${ }^{1}$ Department of Physics and Applied Mathematics, University of Navarra, Pamplona, Spain \\ ${ }^{2}$ PMMH/ESPCI \& CNRS UMR 7636, Universités Paris 6 \& Paris 7, 10 rue Vauquelin, 75005 Paris, France \\ ${ }^{3}$ Service des Basses Températures, INAC, CEA-Grenoble \& Université Joseph Fourier, Grenoble, France \\ (Received 7 November 2012; revised manuscript received 13 October 2013; published 2 January 2014)
}

\begin{abstract}
We describe the evolution of a water drop saturated with $\mathrm{NaCl}$ and the growth of pure water droplets in a breath figure pattern $(\mathrm{BF})$ condensing around it. This salty drop acts as a humidity sink, inhibiting the BF inside a ring at a distance $r=\delta$ from the sink center and slowing down BF growth outside the ring. The initial salty drop is taken either from a salt-saturated solution (type I experiment) or by placing an $\mathrm{NaCl}$ crystal on the substrate (type II experiment). The results are similar, provided that the initial time for type II evolution is taken at the end of the crystal dissolution. The evolution of the salty drop radius $R$ is deduced from the establishment of a three-dimensional hyperbolic concentration profile around the salty drop. This profile scales with $r / \delta$. Accounting for the salt concentration decrease with salty drop growth, $R$ is seen to grow as $t^{5}$. In the region $r>\delta$, water droplets nucleate and grow. The rate of evolution of the water droplets at constant $r / \delta$ can be used to determine the local water pressure. The corresponding data reasonably agree with a hyperbolic water vapor profile around the salty drop. These results can be applied to the growth of BF patterns to determine whether hyperbolic or linear water vapor profiles apply.
\end{abstract}

DOI: 10.1103/PhysRevE.89.012402

PACS number(s): 68.08.Bc, 64.70.fm, 47.85.Np

\section{INTRODUCTION}

When water vapor condenses on a substrate, a droplet pattern usually known as a breath figure (BF) emerges. This phenomenon is ubiquitous in nature, as in the case of dew on plants, and plays an important role in many areas of fundamental and applied sciences. Some examples are textured surfaces [1], superhydrophobic surfaces [2], dew formation [3], and surfaces for improving heat transfer [4]. For these applications a detailed knowledge of nucleation and droplet growth is critical. The preliminary research on BFs dates back to the work done by Aitken, Rayleigh, and Baker during the years from 1893 to 1922 [5-7]. Many fundamental laws of nucleation and growth of BFs have been identified based on Beysens' group studies [3], where very characteristic regimes have been evidenced. After an initial nucleation on surface defects, the growth of isolated droplets proceeds as $t^{1 / 3}(t$ is time). Growth then follows a self-similar growth behavior $(\sim t)$ induced by regular droplet coalescence, with constant drop surface coverage. In a further, and not well understood, final stage, new droplets nucleate between large drops, following the same growth behavior just described.

Individual droplets show a zone of influence, an area surrounding the drop within which it incorporates all molecules. The growing drop is a sink for molecules, so that the nearsurface concentration of molecules within its zone of influence is reduced and the probability that new drop nuclei will form is reduced or eliminated.

Although the water vapor concentration profile is the key parameter that controls growth of the drops, to date there are no measurements reported of this quantity. We

\footnotetext{
*Present address: Instituto de Investigaciones en Materiales, UNAM. Cd. Universitaria, Del. Coyoacán, CP 04510, México DF, México.

†wens@unav.es
}

address this problem in this article by evaluating indirectly the concentration profile from the evolution of a water drop saturated with $\mathrm{NaCl}$ (from a solution or from condensation on a crystal), which acts as a controlled humidity sink, and from the evolution of the BF pattern formed around it.

The paper is organized as follows. In Sec. II we provide a critical review of current theories concerning the growth of a single drop or an assembly of drops on a surface. Our experimental methods are given in Sec. III, followed in Sec. IV by our observations. In Secs. V and VI, the evolution of the salty drop and the region of inhibited condensation around it are discussed. In Sec. VII the evolution of BFs are analyzed, and in Sec. VIII the water concentration profile around the salty drop is evaluated. The results are then generalized to the growth of a drop pattern on a surface in Sec. IX.

\section{BACKGROUND}

\section{A. General}

In order to describe the growth mechanism of a single drop, a number of analytical models have been developed. Let us consider an immobile drop (radius $R$ ) that grows by incorporation of the diffusing water vapor molecules (monomers) around it. The concentration of monomers $n(r, t)$ must obey the following equation ( $t$ is time and $r$ is the distance to the drop center):

$$
\frac{\partial n}{\partial t}=D_{0} \Delta n
$$

Here $D_{0}$ is the diffusion coefficient of the monomers. The drop radius evolution follows a growth equation, where $j$ is the flux of monomers per surface area and $V$ is the drop volume:

$$
\frac{d V}{d t}=\int j d S
$$


The monomer flux is expressed as

$$
j=\left.D_{0} \frac{\partial n}{\partial r}\right|_{R} .
$$

The problem governed by Eqs. (1) and (2) is known as a Stefan problem with a moving boundary at $r=R(t)$. Analytical solutions are rare. For the present problem one assumes a growth slow enough, as in Eq. (1), such that the time dependence of $n$ can be neglected (a quasistatic approximation). Thus Eq. (1) reduces to the following Laplace equation:

$$
\triangle n=0 .
$$

Its solution has to fulfill the boundary conditions. At the drop surface the concentration $n_{0}$ corresponds to the saturation pressure. At infinity is assumed either a constant monomer concentration $n_{\infty}$, corresponding to the supersaturation, or a constant flux of monomers.

\section{B. 3D gradient}

For a single sessile drop on a surface kept at constant temperature, a simple way to solve the problem is to assume an inverse process to evaporation. This evaluation has been performed by Picknett and Bexon [8] and Sokuler et al. [9]. It is implicitly assumed that the probability of incorporating the monomers is uniform on the drop surface, which means that the latent heat of condensation is uniformly removed. Since the top of the drop is further from the surface than the perimeter region, such an assumption is true only for films or drops with low contact angle [10], or when strong convective flows, triggered by buoyancy or thermocapillary forces, are present.

In a 3D space, Eq. (4) has a hyperbolic solution:

$$
n=n_{\infty}-\left(n_{\infty}-n_{0}\right) \frac{R}{r},
$$

with boundary conditions $n(r=0)=n_{0}$ and $n(r \rightarrow \infty)=$ $n_{\infty}$

The following growth law then can be derived from Eq. (2):

$$
R^{2} \frac{d R}{d t}=R^{2} D_{0} \frac{n_{\infty}-n_{0}}{R} .
$$

The integration of this equation gives the classical evolution:

$$
R \sim t^{1 / 2} .
$$

\section{2D gradient}

For near hemispherical drops in the absence of internal convection, the temperature gradient between the drop surface and the substrate is not uniform. The temperature gradient and thus the mass transfer is at a maximum at the perimeter of the drop. Growth should then mostly be due to the incorporation of monomers that diffuse on, or near, the substrate, tending towards the drop perimeter. A two-dimensional (2D) diffusive flux would thus be the dominant growth mechanism. The result is a quasi-2D water concentration gradient where the role of the diffusion of monomers on or close to the surface dominates the process.
Equation (1) can be written, in the quasistatic approximation, as

$$
\frac{\partial^{2} n}{\partial r^{2}}+\frac{1}{r} \frac{\partial n}{\partial r}=0 .
$$

By assuming a constant incoming flux $\Phi_{\infty}$ of monomers at infinity,

$$
2 \pi\left(r D_{0} \frac{\partial n}{\partial r}\right)_{\infty}=\Phi_{\infty},
$$

Steyer et al. [11] found a logarithmic solution for Eq. (1):

$$
n=A \ln r+B .
$$

Here $A$ and $B$ are determined by the boundary conditions.

Mass conservation makes the flux of monomers at infinity and at the perimeter drop the same. Consequently, the growth equation (2) transforms into

$$
R^{2} \frac{d R}{d t} \propto \Phi_{\infty} .
$$

The growth law that follows is then

$$
R \sim t^{1 / 3}
$$

For the same problem, assuming that around a drop (the "trap") there is a constant concentration of moving tiny droplets ("monomers") at infinity, Krapivsky [12] found a $\ln (t)$ modified growth law:

$$
R \sim[t / \ln (t)]^{1 / 3} .
$$

\section{Hampered growth}

Rogers et al. [13] studied the problem of the pattern of droplets that grow by surface diffusion of water molecules and that interact by coalescence. Coalescence events rescale the pattern and give a constant surface coverage during growth. Drops are thus always separated by a distance of the order of their diameter. The concentration gradient around them can overlap and the drops compete for growth. Rogers et al. used a mean-field approximation, where the competition between neighboring drops was replaced by an "effective medium," with an average concentration $n_{m}$. Around each growing drop a boundary layer with length scale $\xi$ was introduced and indicates the average distance from the perimeter of a drop over which the diffusion field is affected by the presence of the drop. The concentration gradient was hypothesized as being linear around each drop, with a typical length scale $\xi$. The flux of monomers into a droplet is then

$$
\Phi=\frac{D_{0} n_{m}}{\xi} .
$$

The growth laws for $R$ and $\xi$ thus follow:

$$
R \sim t^{1 / 4} ; \quad \xi \sim t^{1 / 2} .
$$

To solve the same problem, Beysens and Knobler [14] and Sokuler et al. [9] treated the array of drops like a homogeneous film with an average thickness of $V /\langle d\rangle^{2}$, with $\langle d\rangle$ as the mean drop spacing. Each individual drop (i) of volume $V_{i} \sim$ $R_{i}^{3}$ between coalescence is assumed to grow like a thin film, with the vapor concentration profile depending on the vertical 
coordinate $z$ only. Mass conservation gives a linear gradient and

$$
V_{i} \sim t ; \quad R_{i} \sim t^{\alpha},
$$

where $\alpha=1 / 3$. When accounting for coalescence events, the mean radius evolution of the droplet pattern is rescaled as $\langle R\rangle \sim t^{3 \alpha} \sim t$, as shown by Viovy et al. [15].

\section{E. Thermal effects}

As noted above in Sec. II C, surface diffusion is favored when the absence of convection produces a steady temperature gradient to settle in the drop. In contrast, volume diffusion should dominate when heat can be uniformly removed from the drop interface, as is the case for a film or for drops with a small contact angle or when convection is present. Convection is triggered by temperature gradients and can originate from buoyancy and/or thermocapillary effects. To evaluate the relevance of the former effect to convection, the Rayleigh number

$$
\mathrm{Ra}=\frac{g \beta_{p} \triangle T L^{3}}{\nu D_{T}}
$$

is used. Here $\beta_{p}=0.2 \times 10^{-3} \mathrm{~K}^{-1}$ is the thermal expansion of water at constant pressure $p, g=9.81 \mathrm{~m} \mathrm{~s}^{-2}$ is the earth acceleration constant, $\triangle T$ is the temperature difference that triggers the instability, and $L \sim R$ is the typical distance over which $\triangle T$ acts. $v=1.3 \times 10^{-6} \mathrm{~m}^{2} \mathrm{~s}^{-1}$ is the water kinematic viscosity, and $D_{T}=1.4 \times 10^{-7} \mathrm{~m}^{2} \mathrm{~s}^{-1}$ is the water thermal diffusivity. (All numerical data is from [16].) The instability threshold in the classical Rayleigh-Bénard configuration (two infinite parallel plates with temperature difference $\Delta T$ and separated by distance $L$ ) corresponds to the critical Rayleigh number $\mathrm{Ra}_{c} \approx 1700$. For a drop of radius $R \simeq 500 \mu \mathrm{m}$ (the typical size of a salty drop in this work), convection would start only with an enormous temperature difference in the drop $(1200 \mathrm{~K})$. For smaller drops the temperature difference is even higher. Although the temperature gradients in a drop do not fit the classical Rayleigh-Bénard configuration and salty water does not have exactly the same parameter values as pure water, one can nevertheless conclude that buoyancy flows cannot contribute efficiently to the heat exchange in a water drop.

Another source of convection is concerned with thermocapillary flows, where the governing parameter is the Marangoni number:

$$
\mathrm{Ma}=\left(-\frac{d \sigma}{d T}\right) \frac{1}{\eta D_{T}} L \Delta T .
$$

In this expression, $\sigma$ is the variation of the water-air surface tension with temperature $\frac{d \sigma}{d T} \approx-1.5 \times 10^{-3} \mathrm{~N} \mathrm{~m}^{-1} \mathrm{~K}^{-1}$, and $\eta=1.3 \times 10^{-3} \mathrm{~Pa} \mathrm{~s}$ is the water dynamic viscosity. (All numerical data is from [16].) For the same drop radius as above $(500 \mu \mathrm{m}), \mathrm{Ma} \simeq 3 \times 10^{-3} \triangle T$. With the critical Marangoni number $\mathrm{Ma}_{c} \simeq 60$, thermocapillary flows can start for temperature differences as small as $20 \mathrm{mK}$, thus ensuring an efficient heat transfer from the drop interface to the condensing surface and validating a 3D hyperbolic water pressure profile around the drop. Note that $\mathrm{Ma} \sim R$, such that smaller drops can be free of convection. However, heat and thus mass exchange may be still efficient at this small scale.
The fact that the drop is salty does not substantially modify the parameter values ( $\sigma$ and $\frac{d \sigma}{d T}$ ). This modification can be calculated according to the Raoult law with activity coefficients from Ref. [17] and is found to be negligible.

\section{F. Region of inhibited condensation}

When a drop grows, it creates around itself a circular region of lower supersaturation where new droplets cannot nucleate. We call $\delta$ the distance from the center of the sink to the edge of this region of inhibited condensation (RIC). The growing drop acts as a sink for condensing vapor and diffusing monomers. The fact that new droplets cannot nucleate between drops in the self-similar stage of growth is the hallmark of the presence of this water concentration gradient.

Detailed experimental and analytical studies on the dynamics of the BF on solid surfaces have already been performed $[9,11-13,18,19]$. However, the studies of the dynamics of the inhibited condensation regime in the neighborhood of a single growing drop have been quite limited and have only been performed with an $\mathrm{NaCl}$ saturated drop, where the water vapor pressure is $p_{s 0} \simeq 0.76 p_{s}$. Here $p_{s}$ is the saturation pressure for pure water. Leach et al. [20] have observed that for an $\mathrm{NaCl}$ saturated water drop of radius $1.5-2.5 \mathrm{~mm}, \delta / R=7(\Delta T)^{-0.7}$, where $\Delta T$ is the difference of temperature $T$ between the substrate and the condensing vapor. Williams and Blanc [21] observed the RIC around an $\mathrm{NaCl}$-saturated water drop when the substrate temperature $T_{s}$ was varying. They measured a variation of $\delta$ proportional to the supersaturation $p_{s 0}-p_{s}\left(T_{s}\right)$. Schäfle et al. [22] investigated the dependency of $\delta$ on vapor flux and the wettability of the substrate.

All the studies enumerated above provided information about the dependence of the RIC on several physical parameters. However, experimental data is still lacking concerning the evolution of the salty drop and the shape of the water vapor concentration profile around it.

\section{EXPERIMENT AND METHODS}

The experimental setup consists of a hydrophobic substrate within a Delrin condensation chamber. A Peltier cell is used to cool the substrate to $T_{s}=(10 \pm 0.5)^{\circ} \mathrm{C}$ in all the experiments. In order to achieve a controlled atmosphere, four nozzles are used to stream humid nitrogen inside the chamber during the duration of the experiment. The chamber has a window on its upper side to observe the condensation phenomenon. The observation is performed with a reflection microscope. To monitor the temperature, two thermocouples are used. The first thermocouple is attached close to the area of observation, while the other thermocouple is attached to one of the nozzles in order to measure the ambient temperature in the chamber. A $(165 \pm 0.5) \mathrm{mL} / \mathrm{min}$ flux of nitrogen at $100 \%$ relative humidity at room temperature $T=(23 \pm 0.5)^{\circ} \mathrm{C}$ is streamed into the chamber. The flux is controlled with a digital fluxmeter and leads to a laminar shear flow over the substrate [23]. (For more details about the procedure, see [24].) The substrates are of ITO-coated glass, supplied by Visiontek Systems Limited (UK), with dimensions of $0.1 \times 1.7 \times 1.8 \mathrm{~cm}^{3}$. These substrates, as received from the supplier, are cleaned with ultrapure water in an ultrasonic 
(a)

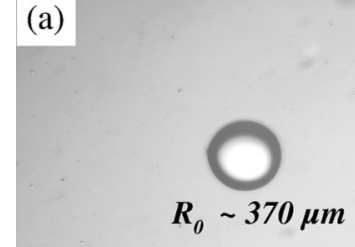

(c)

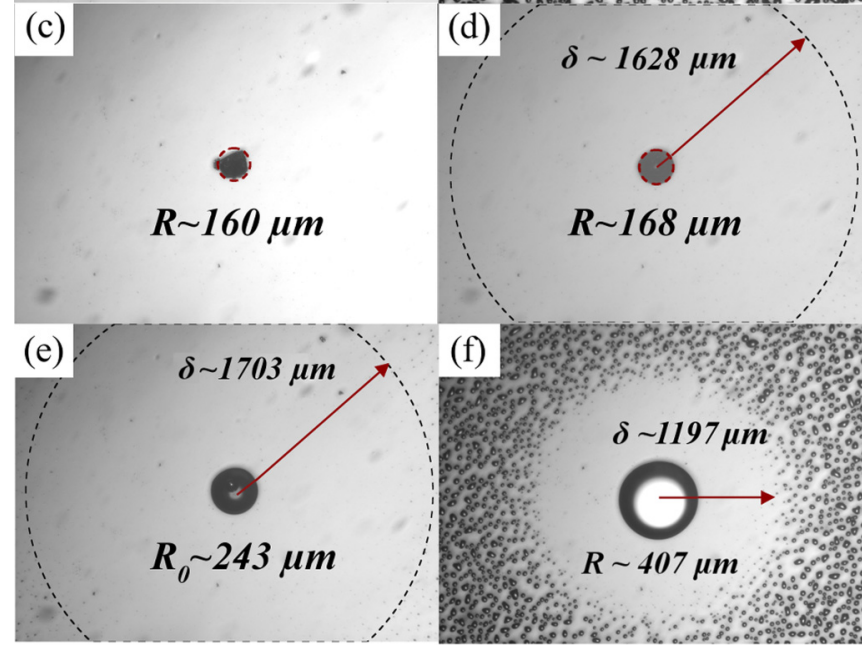

FIG. 1. (Color online) Type-I (a), (b) and type-II (c)-(f) snapshots of the experiment. (a) Time $t=0$. (b) $t=2000 \mathrm{~s}$ with the $\mathrm{BF}$ around the RIC. (c) At $t=0 \mathrm{~s}$ an $\mathrm{NaCl}$ crystal is placed on the substrate. (d) Even the crystal has not been totally dissolved; BF condensation is visible at $t=125 \mathrm{~s}$. (e) The crystal becomes a drop of salt saturated solution and $\mathrm{BF}$ is commencing to appear on the observable area $(t=250 \mathrm{~s})$. A small bubble of trapped air is visible. (f) At $t=2000 \mathrm{~s}$, the stage of the system is comparable to (b).

bath, rinsed several times with ultrapure water, and dried with pure nitrogen. The advancing and receding contact angles are measured by the sessile drop method. For pure water they are $\theta_{A}=(93 \pm 2)^{\circ}$ and $\theta_{R}=(57 \pm 2)^{\circ}$, respectively. The advancing and receding contact angles for a salty drop saturated with $\mathrm{NaCl}$ are $\theta_{A}=(92 \pm 2)^{\circ}$ and $\theta_{R}=(61 \pm 2)^{\circ}$, respectively.

Two kinds of experiments were performed for this study. In the first type of experiment (I), a water drop saturated with $\mathrm{NaCl}$ of submillimeter size is placed on the substrate [see Fig. 1(a)], $R_{0}=(370 \pm 7) \mu \mathrm{m}$. The solution was made according $[25,26]$. At standard temperature and pressure in $100 \mathrm{~g}$ of water, $37.5 \mathrm{~g}$ of sodium chloride was diluted in order to achieve a salt-saturated solution at $T_{s}=10^{\circ} \mathrm{C}$. Once the humid nitrogen is directed to the drop and water vapor condensation starts, the salt concentration decreases in the drop. In the second type of experiment (II), a small $\mathrm{NaCl}$ crystal is placed at $t=0 \mathrm{~s}$ on the substrate. In Fig. 1(c) the apparent radius is $160 \pm 8 \mu \mathrm{m}$. The crystal incorporates water vapor from the surrounding atmosphere and dissolves progressively into the condensed water. In Fig. 1(e) the process of completely dissolving the crystal lasts about $250 \mathrm{~s}$, and the drop radius is then $R_{0}=(243 \pm 12) \mu \mathrm{m}$, corresponding to the salt saturation, as for the drop in the type I experiment. Note that in the dissolution process, air is trapped in the drop and appears as a tiny bubble that progressively dissolves into the salty water. (For both experiments, the time origin for condensation is determined within $5 \mathrm{~s}$.)

A complementary metal-oxide-semiconductor (CMOS) camera is attached to the microscope to periodically acquire images frames every $5 \mathrm{~s}$. Because the advancing contact angles are $\simeq 90^{\circ}$, the $2 \mathrm{D}$ projection of the water and salty drops as seen in these images also represents the drop perimeter. Our setup allows a straightforward image analysis to be made via homemade routines written in OCTAVE and FORTRAN.

To determine $\delta$, we use the position of the salty drop and its size to transform the picture from Cartesian to polar coordinates. $\delta$ is then accurately determined by averaging, in all directions, the distance between the center of the salty drop and the place where BF starts. We also determine the radius $\rho_{i}$ of each BF droplet $(i)$ from their surface areas. The surface area measurements are taken in annuli of width $\Delta=36 \mu \mathrm{m}$.

\section{OBSERVATIONS}

In the type-I experiment, the BF condensation pattern, and thus the region of inhibited condensation, appears soon (around $35 \mathrm{~s}$ ) after the stream of humid $\mathrm{N}_{2}$ is applied onto the observable area. Figure 1(b) shows the BF at 2000 s. In this typical experiment, the initial value for the salty drop radius is $R_{0}=(369 \pm 7) \mu \mathrm{m}$ [Fig. 1(a)]. The evolutions of the drop radius $R$ and the RIC length $\delta$ are shown in Fig. 2(a). The drop radius evolution can be fitted to the following power law, with $\tau$ and $a$ as adjustable parameters:

$$
R=R_{0}\left(1+\frac{t}{\tau}\right)^{1 / a}
$$

with $R_{0}$ imposed to $369.5 \mu \mathrm{m}$, we obtain $a=5.3 \pm 0.01$ and $\tau=(1000 \pm 4) \mathrm{s}$ (error: one standard deviation).

For the type-II experiment, the BF condensation phenomenon appears around $t=125 \mathrm{~s}$ after the stream of humid $\mathrm{N}_{2}$ is applied. At $t=250 \mathrm{~s}$ the crystal is totally dissolved and the RIC is observed. Further evolution is similar to the type-I experiment [Figs. 1(e)-1(f)]. The evolution of $R$ and $\delta$ for this experiment is shown in Fig. 2(b). We took as the initial time the instant when full dissolution of the crystal is observed. The drop radius fits well with Eq. (19) with $R_{0}$ as $310.9 \mu \mathrm{m}$, giving $a=4.98 \pm 0.01$ and $\tau=(300 \pm 2) \mathrm{s}$.

The vapor pressure far from the salty drop (that is, at the border of the hydrodynamic boundary layer where air flow velocity reaches zero) is $p_{\infty}=(2.8 \pm 0.3) \mathrm{kPa}$, which corresponds to the saturation at $T=(23.0 \pm 0.5){ }^{\circ} \mathrm{C}$. It is worth noting that the RIC corresponds to the place where supersaturation reaches the limit for droplet nucleation. On a purely hydrophilic substrate, nucleation corresponds to the saturation pressure $p_{s}\left(=1.2 \mathrm{kPa}\right.$ for $\left.T_{s}=10{ }^{\circ} \mathrm{C}\right)$. On the hydrophobic substrate used here, a temperature difference of $\simeq 1.5^{\circ} \mathrm{C}$ ) with respect to room temperature is needed to observe nucleation of water, corresponding to a supersaturation $\Delta p$ $(\simeq 0.25 \mathrm{kPa})$ [27]. This corresponds to the water vapor pressure $p_{0}=p_{s}+\Delta p(\simeq 1.45 \mathrm{kPa})$. Note also that droplets preferentially form on nucleation sites, the density of which are not controlled and can vary on the substrate. Thus, a larger uncertainty results on $\delta$ than on $R$. A schematic water pressure profile around the salty drop is shown in Fig. 3. 

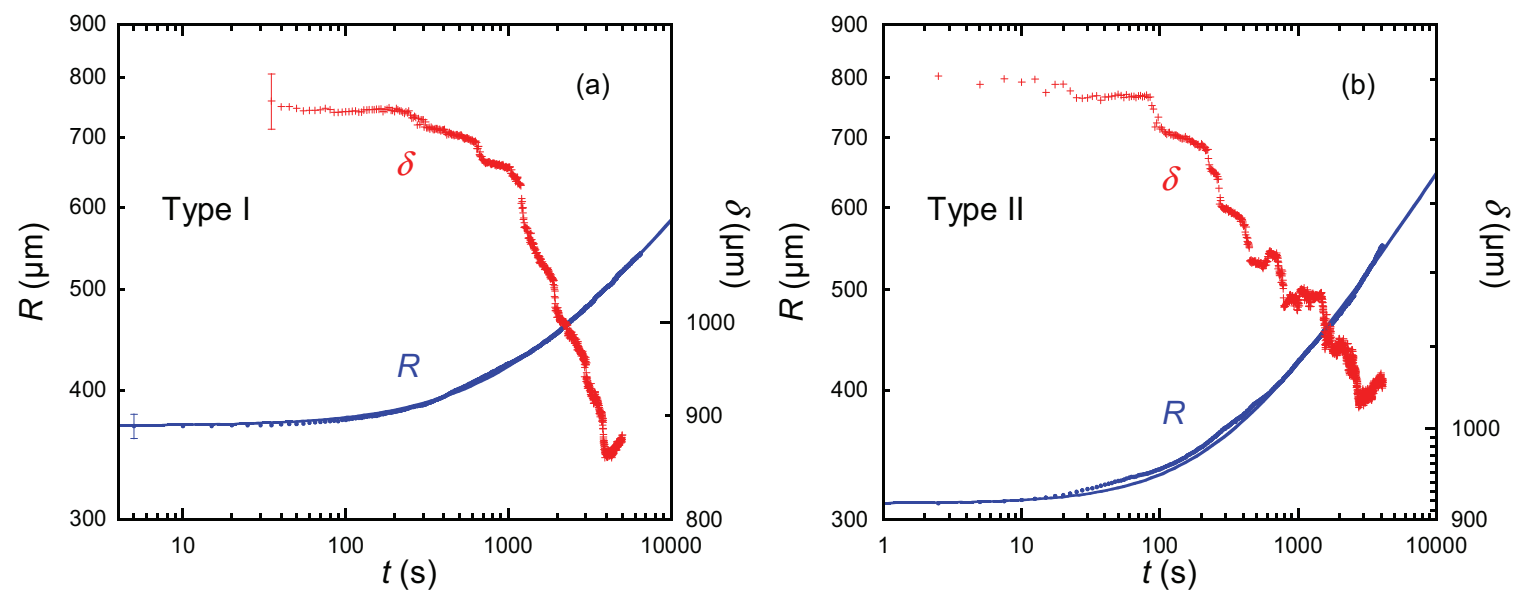

FIG. 2. (Color online) Evolution of $R$ and $\delta$ for (a) a typical experiment of type I and (b) a typical experiment of type II. The solid line is a fit of $R$ to Eq. (19).

\section{SALTY DROP EVOLUTION}

The salty drop grows by diffusion and incorporation of monomers. Figure 4 shows a schematic of the process. As discussed in Sec. II E, a 3D gradient forms around the drop in a diffusion layer above the substrate. The monomer concentration at infinity corresponds to the supersaturation pressure $\left(p_{\infty}\right)$. The saturation water pressure of the salty drop $p_{s s}$ varies during the drop growth due to water condensation. Initially, the water vapor pressure $p_{s s}=p_{s 0}\left(\simeq 0.76 p_{s} \simeq 0.91 \mathrm{kPa}\right)$, corresponding to salt saturation. At late times it reaches the value for pure water, $p_{s s}=p_{s}\left(=1.2 \mathrm{kPa}\right.$ for $\left.T_{s}=10{ }^{\circ} \mathrm{C}\right)$ (Fig. 3).

The model that should apply, in the quasi-static approximation, is thus the 3D model, which leads to a hyperbolic concentration profile [Eq. (5)] with the following boundary conditions (in units of water vapor pressure):

$$
\begin{gathered}
r=R, \quad p=p_{s s} \\
r \rightarrow \infty, \quad p \rightarrow p_{\infty} .
\end{gathered}
$$

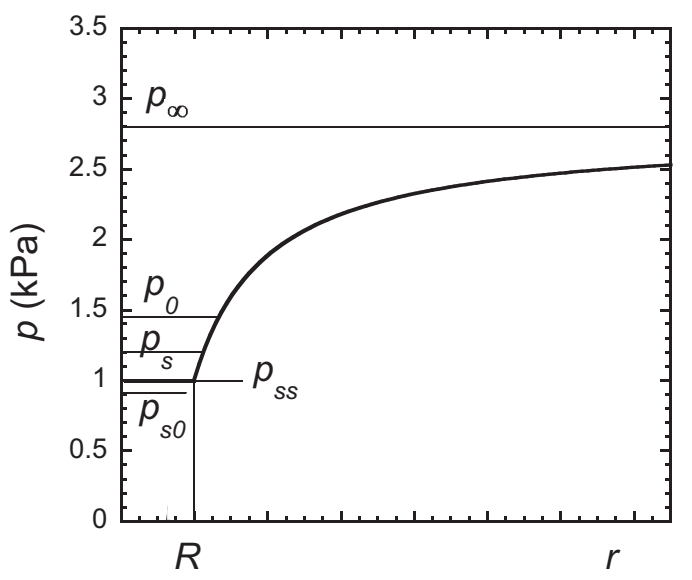

FIG. 3. Water vapor concentration profile as a function of the distance $r$ to the salty drop center (schematics). $p_{s s}$ is the salty drop water saturation pressure, and varies during growth from the value at salt saturation $p_{s 0}\left(\simeq 0.76 p_{s} \simeq 0.91 \mathrm{kPa}\right)$ to the value for pure water $p_{s}\left(=1.2 \mathrm{kPa}\right.$ for $\left.T_{s}=10^{\circ} \mathrm{C}\right)$.
The solution is

$$
p=p_{\infty}+\left(p_{s s}-p_{\infty}\right) \frac{R}{r}
$$

The growth equation is thus as follows:

$$
\left.R^{2} \frac{d R}{d t} \sim R^{2} \frac{\partial p}{\partial r}\right|_{R}=R^{2} \frac{\left(p_{\infty}-p_{s s}\right)}{R} .
$$

During growth, the salt concentration (and thus, the local supersaturation) decreases. This in turn modifies the water pressure gradient. We use the Raoult law to evaluate the variation of the saturation pressure with the salty drop radius. A detailed calculation is given in the Appendix and leads to the approximate variation

$$
p_{s}-p_{s s} \simeq\left(p_{s}-p_{s 0}\right)\left[\frac{R_{0}}{R}\right]^{b},
$$

with the exponent $b \simeq 3.3$. In the limit of small concentration, a simplified expression corresponds to $b=3$ :

$$
p_{s}-p_{s s} \simeq\left(p_{s}-p_{s 0}\right)\left[\frac{R_{0}}{R}\right]^{3} .
$$

As $b$ is not significantly different in both Eqs. (24) and (25) even for small times, we will make, for sake of simplicity, $b=3$ in the following and use Eq. (25). Equation (23) then

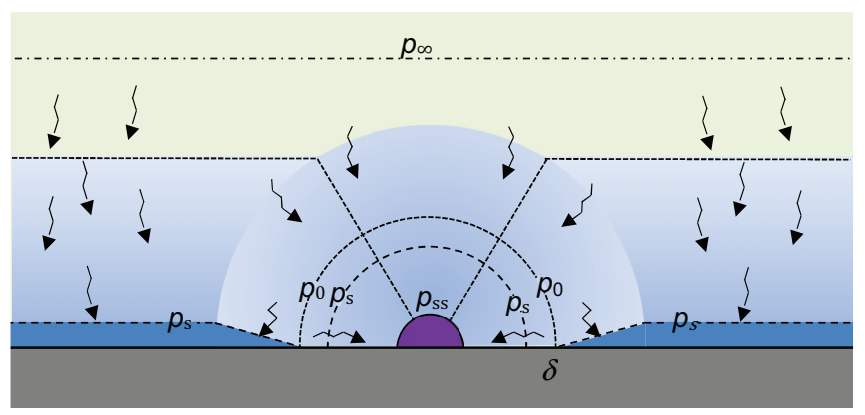

FIG. 4. (Color online) Schematic of the condensation process (see text). 
becomes

$$
\begin{aligned}
R \frac{d R}{d t} & \sim\left(p_{\infty}-p_{s}\right)+\left(p_{s}-p_{s s}\right) \\
& =\left(p_{\infty}-p_{s}\right)+\left(p_{s}-p_{s 0}\right)\left[\frac{R_{0}}{R}\right]^{3} .
\end{aligned}
$$

The standard way to solve this equation is to invert it and solve $t(R)$. It easily reduces to the integral of $R /\left(R^{3}+\right.$ constant), which can be put in terms of the arctan and log of algebraic functions. The solution can be found in classical handbooks, cf. e.g., [28]. Although it is not possible to invert the result analytically to obtain the evolution $R(t)$, one can consider the asymptotic regimes.

At long times, the salty drop becomes highly diluted and the first term in the equation is dominant. The depletion zone is of the same order as in pure water. Equation (26) can be written

$$
R \frac{d R}{d t} \sim\left(p_{\infty}-p_{s}\right)
$$

This reflects the classical evolution of Eq. (7), where $R \sim t^{1 / 2}$ is recovered.

When the drop is close to salt saturation, the second term $R^{-3}$ in Eq. (26) is more relevant than the first term. Equation (26) becomes

$$
R \frac{d R}{d t} \sim\left(p_{s}-p_{s 0}\right)\left[\frac{R_{0}}{R}\right]^{3},
$$

the solution of which is

$$
R^{5}=R_{0}^{5}+B t
$$

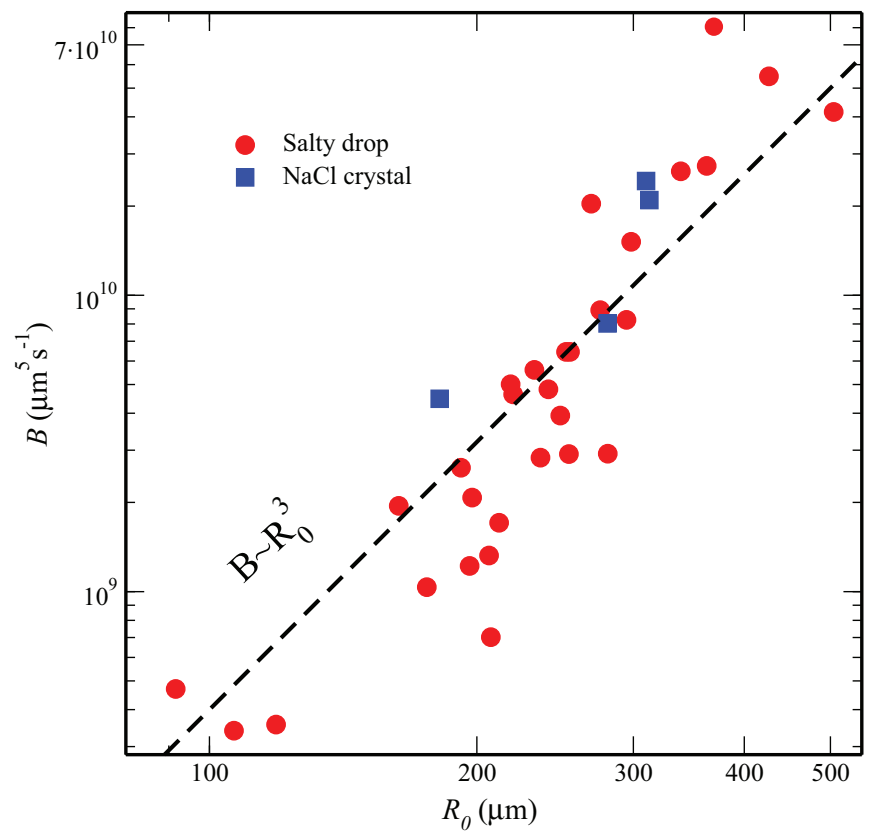

FIG. 5. (Color online) Amplitude $B$ [see Eq. (29)] for several experimental measurement sets with different initial saturated salty drop radius $R_{0}$. The dashed line is a power law with an exponent 3 . Circles (red) correspond to type-I experiments and squares (blue) to type-II experiments.

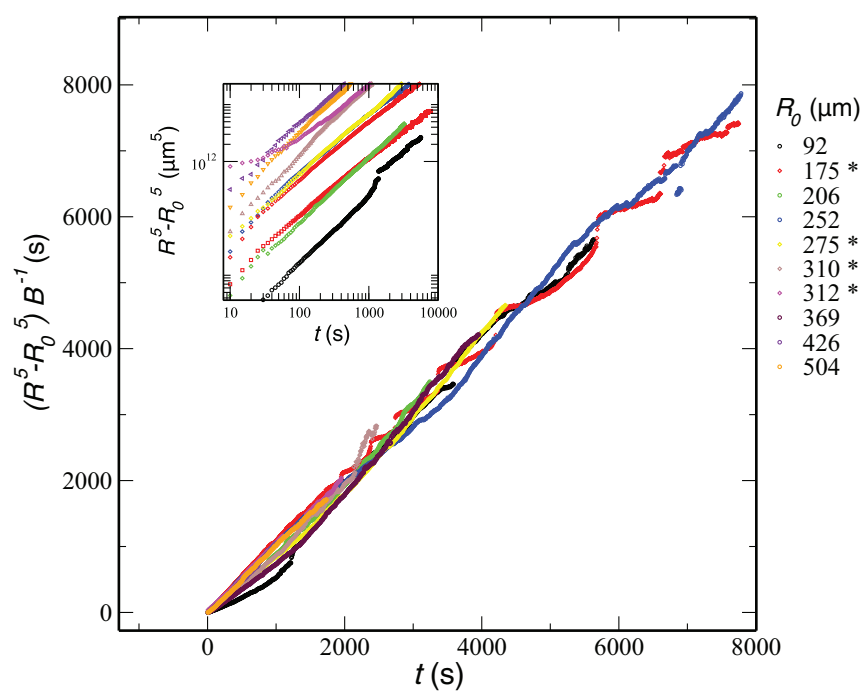

FIG. 6. (Color online) Inset: linear evolution of $R^{5}-R_{0}^{5}$. When rescaled by the corresponding $B^{-1}$ values [Eq. (29)], the curves collapse to one single linear growth law. Stars in the values of $R_{0}$ (right column) correspond to type-II experiments.

where

$$
B \propto R_{0}^{3} .
$$

If we take $a=5$ and $\tau=R_{0}^{5} / B$ in Eq. (19), we recover Eq. (29). In Fig. 5 the variation of $B$ with respect to $R_{0}$ does indeed follow a power law with an exponent 3 , in accordance with Eq. (30). The different sets of data also fall on one single curve when properly scaled: see Fig. 6, where the evolution of $\left(R^{5}-R_{0}^{5}\right) / B$ is reported.

\section{REGION OF INHIBITED CONDENSATION}

The RIC corresponds to the place where supersaturation reaches the limit that permits droplet nucleation, that is, $p=$ $p_{0}$. From the hyperbolic profile Eq. (22) the above condition can be written as

$$
p_{0}=p_{\infty}+\left(p_{s s}-p_{\infty}\right) \frac{R}{\delta} .
$$

Using Eq. (25), it follows that

$$
\frac{\delta}{R}=\frac{p_{\infty}-p_{s}}{p_{\infty}-p_{0}}+\frac{p_{s}-p_{s 0}}{p_{\infty}-p_{0}}\left(\frac{R}{R_{0}}\right)^{-3} .
$$

The variation of $\delta / R$ with respect to $R / R_{0}$ is shown in Fig. 7 for typical experiments of type I and II. When fitted to Eq. (32) as $\delta / R=C+D\left(R / R_{0}\right)^{-3}$ with $C$ and $D$ as adjustable parameters, the general variation is reproduced for both experiments. Both variations are similar; however, they show difference in the amplitude. The expected values are $C=$ $\frac{p_{\infty}-p_{s}}{p_{\infty}-p_{0}}=1.185$ and $D=\frac{p_{s}-p_{s 0}}{p_{\infty}-p_{0}}=0.21$. The parameter values that are found are comparable for both types I and II concerning $D \approx 3.3$ (type I) and $\approx 3.8$ (type II), but larger than expected. They differ, however, for $C \approx 0.5$ (type I) and $\approx 1.2$ (type II), also in disagreement with the expected values. The main source of discrepancy is the high sensitivity of the determination of $\delta$ with the density and chemical characteristics ( $p_{0}$ value) of the 


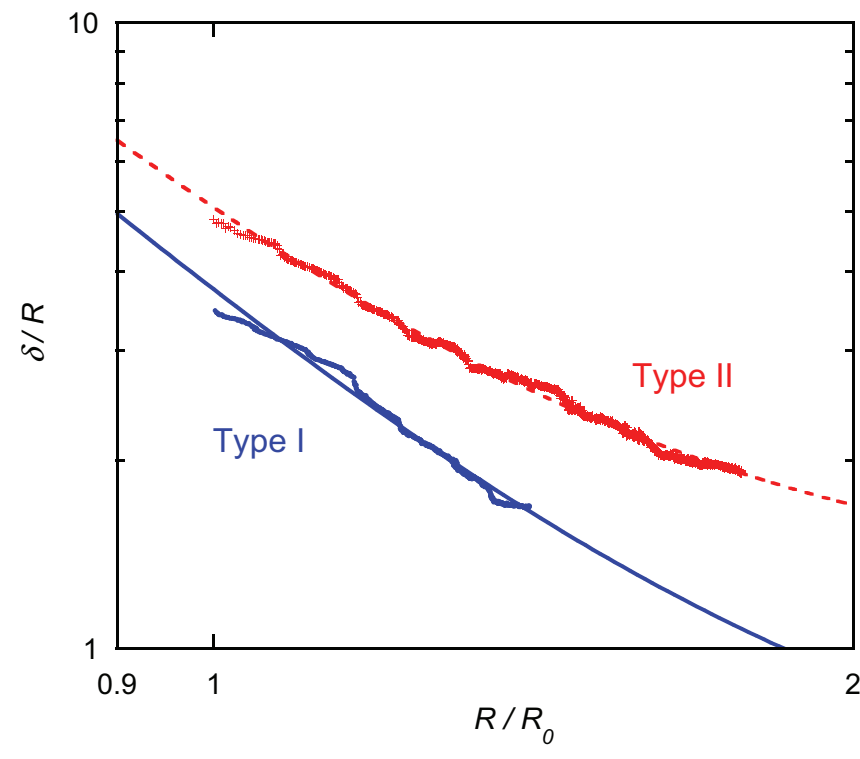

FIG. 7. (Color online) Variation of $\delta / R$ with respect to $R / R_{0}$ (log-log plot) for the typical experiments shown in Fig. 2. The curves are fits to Eq. (32).

nucleation sites, a parameter which remains uncontrolled and can vary with each experimental set and sample.

Returning to the vapor concentration profile Eq. (22), we obtain

$$
p=p_{\infty}-\left(p_{\infty}-p_{0}\right) \frac{\delta}{r} .
$$

As a result, the profile around the salty drop should scale with $\delta$. This is an important result that will be used in the next section to experimentally determine the vapor concentration profile from the growth rate of $\mathrm{BF}$ droplets in the region $r>\delta$.

\section{BREATH FIGURES GROWTH LAWS}

The growth law of water droplets outside the RIC depends on the local water supersaturation pressure. In the concentration gradient around the salty drop, a BF drop pattern will nucleate and grow only for $p>p_{0}$, which corresponds to distances $r>\delta$ from the salty drop (Fig. 4). In classical $\mathrm{BF}$ growth, the droplet radius evolution $\rho(t)$ corresponding to various constant supersaturation $\triangle p_{i}=p_{i}-p_{s}$ can be rescaled on a unique curve $\rho\left(t / t_{i}\right)$ with $t_{i} \sim 1 / \triangle p_{i}$ [29]. The droplet evolution in the BF pattern around the salty drop can then be used to determine the local supersaturation profile $p_{i}-p_{s}=p(r)-p_{s}$.

However, a difficulty arises because the above growth laws correspond to a supersaturation that remains constant over time. In contrast, in the present configuration, the droplets evolve according to a time-dependent local supersaturation. In other words, supersaturation varies over time at constant $r$. However, based on Eq. (33), the concentration profile should scale with $r / \delta$. The growth laws of BFs at given $r / \delta$ should correspond to constant supersaturation levels. The mean radius $\langle\rho\rangle$ of BF water droplets is then measured around the salty drop at distances $r$ in the range $r>\delta$ in concentric annuli of thickness $\Delta$. And in fact, in Fig. 8 (a) we see that the evolution at
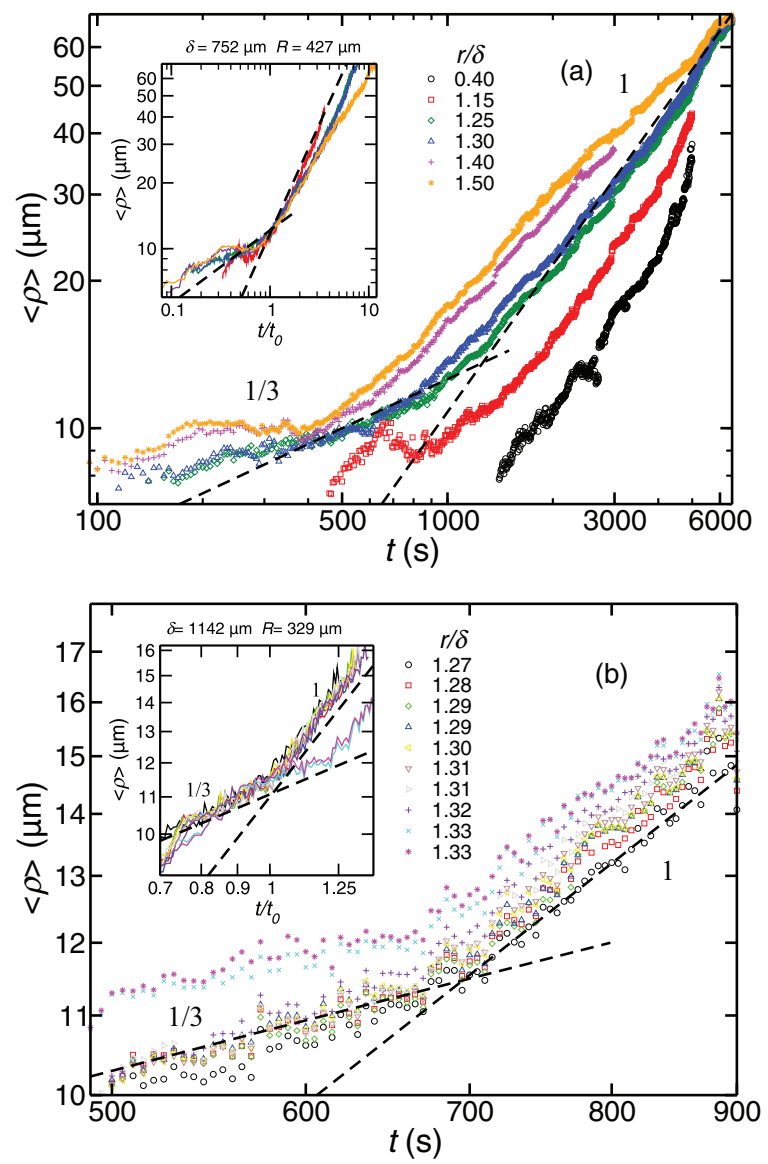

FIG. 8. (Color online) Evolution of water droplet mean radius $\langle\rho\rangle$ for several ratios $r / \delta$ corresponding to the type-I experiment (a) and type-II experiment (b) from Fig. 2. The initial $t^{1 / 3}$ and final $t^{1}$ growth laws are clearly visible (dashed lines). The insets show the same data with time rescaled by the onset of nucleation time, $t_{i}$.

constant $r / \delta$ corresponds to what is currently observed for BF growth at constant supersaturation: the initial $t^{1 / 3}$ growth law is followed, when droplet coalescence dominates the process by a $t$ growth. The various $t^{1 / 3}$ growth laws can be rescaled by times $\left(t_{i}\right)$ such that they collapse on the same $\left(t / t_{i}\right)^{1 / 3}$ growth stage (Fig. 8). Note that the further $t^{1}$ growth laws do not fit the rescaling well. This is due to the fact that the crossover time to the coalescence-dominated stage depends on the drop interdistance and then on the initial density of nucleation sites. The latter changes with each experimental set and sample, and remains mostly uncontrolled. Nevertheless, from the above rescaling by $t_{i}$ one obtains

$$
\left[p(r / \delta)-p_{s}\right] \sim 1 / t_{i}
$$

\section{CONCENTRATION PROFILE}

The scaling or visibility inverse times $1 / t_{i}$ are thus then proportional to the local supersaturation $p(r / \delta)-p_{s}$ :

$$
1 / t_{i}=E\left[1-\frac{p_{\infty}-p_{0}}{p_{\infty}-p_{s}} \frac{\delta}{r}\right],
$$

where $E$ is a proportionality constant. 


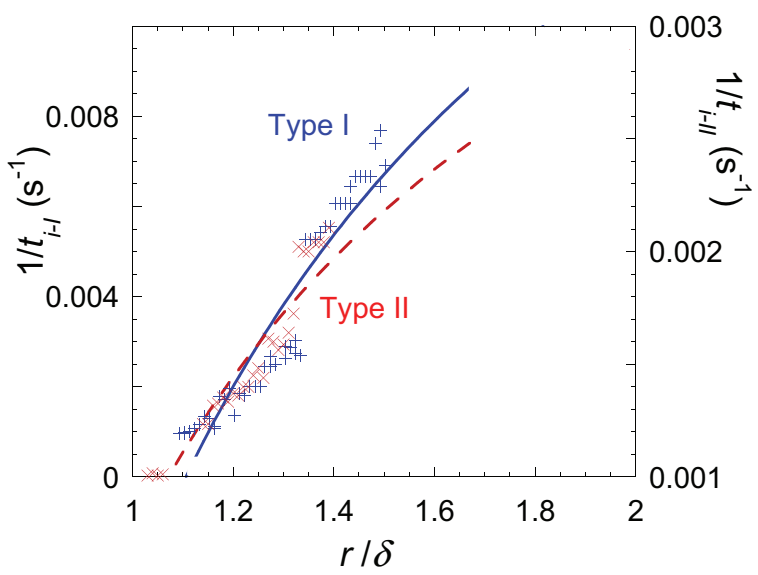

FIG. 9. (Color online) Water concentration profile (in units of inverse rescaling time $t_{i}$ ) with respect to $r / \delta$ corresponding to the experiments of Fig. 2. The curves are fits to Eq. (35). Solid line and + symbols (blue) refer to type-I experiments while dashed line and $\times$ symbols (red) refer to type-II experiments.

We make the assumption (which we hold to be reasonable) that the growth of the BF droplets does not appreciably modify the water vapor profile around the salty drop. The $1 / t_{i}$ data should therefore follow a hyperbolic variation with respect to $r / \delta$. In Fig. 9 the $1 / t_{i}$ data fits

$$
1 / t_{i}=E[1-F(\delta / r)] \text {. }
$$

The data fits the above variation reasonably well, with $E=0.028 \pm 0.002$ and $F=0.9 \pm 0.01$ (type I, one standard deviation), $E=0.044 \pm 0.001$ and $F=1.16 \pm 0.03$ (type II, one standard deviation). The $F$ value also agrees well between experiments types I and II, and the expected value for $\frac{p_{\infty}-p_{0}}{p_{\infty}-p_{s}} \simeq 0.71$. The main source of errors is the uncontrolled density of nucleation sites, as already noted. As a matter of fact, the depletion zone is observed to shrink with time in a noncontinuous manner, leading sometimes to a stepwise evolution, as can be seen in Fig. 2. It then results a kind of discontinuity, as can be noted in Fig. 9.

\section{WATER DROPS}

The characterization of a salty drop we have developed in this article can be applied both to the case of a single water drop as well as to a pattern of water drops.

The concentration profile around a pure water drop of radius $\rho$ can be deduced from Eq. (22), where $p_{s s}$ has to be replaced by $p_{s}$ and $R$ by $\rho$ :

$$
p=p_{\infty}+\left(p_{s}-p_{\infty}\right) \frac{\rho}{r} .
$$

The definition of $\delta$ corresponds to $p=p_{0}$. This gives

$$
\delta=\rho\left(\frac{p_{\infty}-p_{s}}{p_{\infty}-p_{0}}\right) .
$$

In the present experimental configuration, $\delta \simeq 1.6 \rho$.

Let us now consider a pattern of growing droplets. When the droplet surface coverage $\epsilon^{2}$ is large enough, coalescence events rescale the pattern such that $\epsilon^{2}$ remains constant. The average droplet radius and the mean distance $\langle d\rangle$ between the drop centers $\langle\rho\rangle \sim\langle d\rangle \sim t$. Assuming for the sake of simplicity that the drops are set on a square lattice, we get

$$
\langle d\rangle^{2}=\pi \frac{\langle\rho\rangle^{2}}{\epsilon^{2}} .
$$

A relation can be found between $\epsilon^{2}$ and $\theta$ for a smooth substrate [30]. This corresponds to the competition, during the coalescence of two drops, between the pinning forces at the contact lines (which resist to the drop motion) and the capillary forces (which move the drops). For large $\theta$, the capillary force dominates, and the new drop that results from coalescence has the same geometry as the parent drops, with a small $\epsilon^{2}$. For small $\theta$, the pinning force prevails and the new drop is irregular, with a large $\epsilon^{2}$. This relation can be written as (with $\theta$ in degrees)

$$
\epsilon^{2} \approx 1-\frac{\theta}{200}
$$

From Eq. (39) we get

$$
\langle d\rangle^{2} \approx \frac{\pi}{1-\frac{\theta}{200}}\langle\rho\rangle^{2} .
$$

For the present experiment where $\theta=\theta_{A}=93^{\circ}$, the surface coverage is $\epsilon^{2} \approx 0.54$, and as a result, $\langle d\rangle \approx 2.4\langle\rho\rangle$.

Nucleation is not possible between drops when $\delta<\langle d\rangle$ or

$$
\frac{p_{\infty}-p_{s}}{p_{\infty}-p_{0}}<\left[\frac{\pi}{(1-\theta / 200)}\right]^{1 / 2} .
$$

It is the case of the present experimental situation, where $\delta / \rho \simeq$ $1.6<2.4 \approx\langle d\rangle / \rho$ and nucleation of new droplets cannot take place. However, in the development of a BF pattern, at long times when drops are large, a new stage of growth is observed when nucleation of a new BF pattern starts between these large drops [3]. This renucleation phenomenon corresponds to the failure of the assumption of a mean linear vapor concentration profile directed perpendicular to the surface for the BF growth (see above, Sec. IID). In other words, the vapor profile of each drop no longer overlaps and nucleation of new droplets can occur between them. Equation (42) above thus gives the condition for the onset of this late stage of growth.

\section{CONCLUDING REMARKS}

The results reported above are concerned with the growth of a salty drop by water vapor condensation. We anticipate that detailed knowledge of this process and the determination of the growth laws for the drop radius and the region of inhibited condensation will be useful for any process where water and hygroscopic materials are involved.

From a more fundamental point of view, the interplay of the vapor profile around a salty drop and an assembly of water droplets makes apparent the different vapor gradients that can occur during dropwise condensation and their interplay when a droplet pattern is present. In particular, the growth rate data of the assembly of BF water droplets around the salty drop validate the presence of a hyperbolic profile around the salty drop and a mean linear profile above the water droplet pattern. The manifestation of the last stage of BF growth, when new tiny droplets can nucleate and grow between the large drops of the former self-similar stage, is well explained 
in this framework. It corresponds to the situation where the water vapor concentration profiles around the drops no longer overlap, which implies the failure of the assumption of a mean linear profile above the drop pattern. The time when this change occurs depends on supersaturation and the water-substrate contact angle.

\section{ACKNOWLEDGMENTS}

We gratefully acknowledge the comments of A. Yethiraj and V. Nikolayev, after a critical reading of the manuscript, and Erik Norvelle for carefully reading the manuscript. We thank the anonymous reviewers for their useful comments. This work was partly supported by the Spanish MEC (Grant No. FIS2011-24642) and by Departamento de Educación (Gobierno de Navarra). R.D.N. acknowledges the support of a Marie Curie International Incoming Fellowship (MCIIF) within the 7th European Community Framework Program, and J.G.C. acknowledges financial support from the "Asociación de Amigos de la Universidad de Navarra."

\section{APPENDIX}

The original Raoult law in our system is

$$
p_{s s}=p_{s} \frac{n_{\mathrm{H}_{2} \mathrm{O}}}{n_{\mathrm{H}_{2} \mathrm{O}}+n_{\mathrm{NaCl}}},
$$

where $n_{\mathrm{NaCl}}$ is the number of moles of salt, which is constant in an experiment. $n_{\mathrm{H}_{2} \mathrm{O}}$ is the number of moles of water, which depends on the vapor condensation history and on the initial size of the drop. Consequently,

$$
p_{s}-p_{s s}=\frac{n_{\mathrm{NaCl}}}{n_{\mathrm{H}_{2} \mathrm{O}}+n_{\mathrm{NaCl}}} p_{s} .
$$

At the saturated condition (salt in water at $10{ }^{\circ} \mathrm{C}$ ),

$$
p_{s}-p_{s 0}=\frac{n_{\mathrm{NaCl}}}{n_{\mathrm{H}_{2} \mathrm{O}}^{s}+n_{\mathrm{NaCl}}} p_{s},
$$

where $n_{\mathrm{H}_{2} \mathrm{O}}^{s}$ is the number of water moles which are needed to saturate the solution with the specified $n_{\mathrm{NaCl}}$ at $10{ }^{\circ} \mathrm{C}$.

From Eqs. (A2) and (A3) one gets

$$
p_{s}-p_{s s}=\left(p_{s}-p_{s 0}\right) \frac{n_{\mathrm{H}_{2} \mathrm{O}}^{s}+n_{\mathrm{NaCl}}}{n_{\mathrm{H}_{2} \mathrm{O}}+n_{\mathrm{NaCl}}} \text {. }
$$

With $m_{t}, M_{\mathrm{H}_{2} \mathrm{O}}, M_{\mathrm{NaCl}}$ being the total mass of the drop, and the molecular masses of water and salt, respectively, then

$$
m_{t}=M_{\mathrm{H}_{2} \mathrm{O}} n_{\mathrm{H}_{2} \mathrm{O}}+M_{\mathrm{NaCl}} n_{\mathrm{NaCl}},
$$

and in a hemispherical drop (a very good approximation in our experiments),

$$
m_{t}=\frac{2 \pi}{3} R^{3} \rho .
$$

It is worth noting that $\rho=\rho(c, T)$, thus indirectly depending on $R$. In our experiments we are assuming that the drop temperature is constant. Finally, it is possible to obtain the exact expression:

$$
p_{s}-p_{s s}=\left(p_{s}-p_{s 0}\right) \frac{R_{0}^{3} \frac{2 \pi \rho_{s}}{3}-n_{\mathrm{NaCl}}\left(M_{\mathrm{H}_{2} \mathrm{O}}-M_{\mathrm{NaCl}}\right)}{R^{3} \frac{2 \pi \rho}{3}-n_{\mathrm{NaCl}}\left(M_{\mathrm{H}_{2} \mathrm{O}}-M_{\mathrm{NaCl}}\right)} .
$$

Here, the density of the saturated solution $\rho_{s}$ is different from $\rho$, which depends ultimately (but very weakly) on $R$. In our experimental conditions it is very useful to obtain an approximation of Eq. (A7). To do that, we can differentiate Eq. (A2) and obtain after some arithmetics,

$$
\frac{d\left(p_{s}-p_{s s}\right)}{p_{s}-p_{s s}}=-\frac{d n_{\mathrm{H}_{2} \mathrm{O}}}{n_{\mathrm{H}_{2} \mathrm{O}}+n_{\mathrm{NaCl}}} .
$$

By differentiating Eqs. (A5) and (A6), using $\rho=\rho(c)$ where $c=\frac{M_{\mathrm{NaCl}}^{n} \mathrm{NaCl}}{m_{t}}$ is the concentration weight by weight, it is possible to obtain the following:

$$
\frac{d\left(p_{s}-p_{s s}\right)}{p_{s}-p_{s s}}=-3 \frac{d R}{R} \frac{1}{\left[1+c \frac{\rho^{\prime}}{\rho}\right]} \frac{1}{\left[1+\frac{n_{\mathrm{NaCl}}\left(M_{\mathrm{H}_{2} \mathrm{O}}-M_{\mathrm{NaCl}}\right)}{\frac{2}{3} \pi R^{3} \rho}\right]},
$$

where $\rho^{\prime}$ is the derivative of $\rho$ with respect to $c$.

Equation (A9) can be exactly integrated and leads to Eq. (A7). As $\rho$ and $R$ vary very smoothly, $1+c \frac{\rho^{\prime}}{\rho} \approx 1.002$ [31] and $1+\frac{n_{\mathrm{NaCl}}\left(M_{\mathrm{H}_{2} \mathrm{O}}-M_{\mathrm{NaCl}}\right)}{\frac{2}{3} \pi R^{3} \rho} \approx 0.911$ [31]. Then, Eq. (A9) becomes

$$
\frac{d\left(p_{s}-p_{s s}\right)}{p_{s}-p_{s s}} \simeq-3.3 \frac{d R}{R}
$$

which can be integrated to give Eq. (24), with $b \simeq 3.3$.
[1] K. K. Varanasi, M. Hsu, N. Bhate, W. Yang, and T. Deng, Appl. Phys. Lett. 95, 094101 (2009).

[2] C. Dietz, K. Rykaczewski, A. G. Fedorov, and Y. Joshi, Appl. Phys. Lett. 97, 033104 (2010).

[3] D. Beysens, C. R. Acad. Phys. 7, 1082 (2006).

[4] L. Zhong, M. Xuehu, W. Sifang, W. Mingzhe, and L. Xiaonan, Biochem. Eng. J. 156, 546 (2010).

[5] J. Aitken, Proc. R. Soc. Edinburgh 20, 94 (1893).

[6] L. Rayleigh, Nature (London) 86, 416 (1911).
[7] T. Baker, Philos. Mag. Series 6 44, 752 (1922).

[8] R. Picknett and R. Bexon, J. Colloid Interface Sci. 61, 336 (1977).

[9] M. Sokuler, G. Auernhammer, C. Liu, E. Bonaccurso, and H.-J. Butt, Europhys. Lett. 89, 36004 (2010).

[10] C. Parneix, P. Vandoolaeghe, V. S. Nikolayev, D. Quéré, J. Li, and B. Cabane, Phys. Rev. Lett. 105, 266103 (2010).

[11] A. Steyer, P. Guenoun, D. Beysens, and C. M. Knobler, Phys. Rev. A 44, 8271 (1991). 
[12] P. L. Krapivsky, Phys. Rev. E 47, 1199 (1993).

[13] T. M. Rogers, K. R. Elder, and R. C. Desai, Phys. Rev. A 38, 5303 (1988).

[14] D. Beysens and C. M. Knobler, Phys. Rev. Lett. 57, 1433 (1986).

[15] J. L. Viovy, D. Beysens, and C. M. Knobler, Phys. Rev. A 37, 4965 (1988).

[16] CRC Handbook of Chemistry and Physics, 87th ed., edited by D. R. Lide (CRC Press, Boca Raton, FL, 2006).

[17] Z. Li and B. C.-Y. Lu, Chem. Eng. Sci. 56, 2879 (2001).

[18] P. Meakin, Rep. Prog. Phys. 55, 157 (1992).

[19] B. J. Briscoe and K. P. Galvin, J. Phys. D: Appl. Phys. 23, 1265 (1990).

[20] R. N. Leach, F. Stevens, S. C. Langford, and J. T. Dickinson, Langmuir 22, 8864 (2006).

[21] R. Williams and J. Blanc, J. Chem. Phys. 74, 4675 (1981).

[22] C. Schäfle, P. Leiderer, and C. Beschinger, Europhys. Lett. 63, 394 (2003).
[23] J. Guadarrama-Cetina, Ph.D. thesis, University of Navarra, 2013.

[24] R. D. Narhe, W. González-Viñas, and D. A. Beysens, Appl. Surf. Sci. 256, 4930 (2010).

[25] S. Sawamura, N. Egoshi, Y. Setoguchi, and H. Matsuo, Fluid Phase Equilib. 254, 158 (2007).

[26] I. Arias-González, J. Reza, and A. Trejo, J. Chem. Thermodyn. 42, 1386 (2010).

[27] J. Guadarrama-Cetina and W. González-Viñas, Phys. Rev. E 87, 054401 (2013).

[28] S. Lipschutz, M. Spiegel, and J. Liu, Schaum's Outlines: Mathematical Handbook of Formulas and Tables (McGraw-Hill, New York, 2008).

[29] D. Fritter, C. M. Knobler, and D. A. Beysens, Phys. Rev. A 43, 2858 (1991).

[30] H. Zhao and D. Beysens, Langmuir 11, 627 (1995).

[31] Chemist Reference Book, edited by B. P. Nikolskij, O. N. Grigorov, and M. E. Posin et al. (Goskhimizdat, MoscowLeningrad, 1964), Vol. III. 